\title{
Comparação entre as técnicas de quatro tamises e de punção- biópsia hepática, para o diagnóstico da Fasciolose ovina*
}

\section{Comparison among the techniques of four sieves and of hepatic puncture-biopsy, for Fascioliasis ovine's diagnosis}

\author{
Edwin Pile, ${ }^{\star \star}$ Sabrina Ferreira, ${ }^{\star \star \star}$ José Augusto Albuquerque dos Santos, ${ }^{\star \star}$ Paulo Oldemar Scherer ${ }^{\star \star \star \star}$
}

\begin{abstract}
Resumo
É feita a comparação entre a técnica de quatro tamises e a de punção-biópsia hepática, para o diagnóstico da fasciolose ovina. A análise foi feita a partir de exames realizados em 77 amostras coletadas de animais procedentes do Rio Grande do Sul, Brasil. Os resultados demonstraram diferença significativa entre as técnicas analisadas, identificando a técnica de punçãobiópsia hepática como a de maior valor diagnóstico. Entretanto, se discute a dificuldade de sua utilização.
\end{abstract}

Palavras-chave: Fasciola hepatica; fasciolose; diagnóstico.

\begin{abstract}
It is made the comparison between the technique of four sieves and the hepatic puncture-biopsy, for the diagnosis of the ovine fascioliasis. Analysis was made starting from exams accomplished in seventy seven samples collected in the Rio Grande do Sul state, Brazil.. The results showed significant difference among the techniques used, identifying the technique of hepatic puncture-biopsy as the one of larger value diagnosis. However, it discusses the difficulty of its use.
\end{abstract}

Keywords: Fasciola hepatica; fascioliasis; diagnosis.

A presença da fasciolose hepática, hoje amplamente distribuída, é considerada um fator limitante na criação de bovinos e ovinos em vários países do mundo. Os prejuízos econômicos provocados pela mortalidade de animais jovens, pela perda por condenação de fígados em matadouros, na produção de carne, leite e lã e pela diminuição da fertilidade dos rebanhos não são os únicos, pois a fasciolose também pode afetar o homem (Alcaíno et al., 1989).

Ao tentar controlar a doença através de programas estratégicos, nos deparamos com vários problemas que começam no diagnóstico laboratorial. Observa-se, assim, que as numerosas técnicas empregadas no imunodiagnóstico da fasciolose tanto em animais como em humanos, utilizando diferentes preparações antigênicas, demonstram desvantagens que acabam por tirar a sensibilidade dos testes (Berne, 1994). A opção de realizar necrópsias nem sempre é possível, restando apenas as técnicas coproscópicas e as de punção-biópsia. Desta forma, os exames coproscópicos são as formas de diagnóstico mais freqüentes no Brasil. Conseqüentemente, o diagnóstico só é fèito na fase crônica da doença, enquanto nas fases aguda e subaguda o diagnóstico efetivo só é fornecido pelos achados de necrópsia.
Com base nesses dados e observando a possibilidade de expansão da doença (Scherer et al., 1995), decidiu-se comparar duas técnicas de diagnóstico de eficácia comprovada: a técnica de Quatro Tamises e a de Punção-biópsia hepática, recentemente desenvolvida, avaliando, assim, seu valor diagnóstico.

Para realizar-se os trabalhos foram amostrados 77 ovinos, com aproximadamente sete meses de idade, oriundos de vários distritos do município de Santa Vitória do Palmar (SVP) - RS, utilizando a técnica de punção-biópsia descrita por Scherer et al. (1995). Em seguida, amostras de fezes desses animais foram coletadas diretamente da ampola retal. Os exames coprológicos foram realizados através da técnica de Quatro Tamises, seguindo a metodologia descrita por Girão e Ueno (1985). Na histopatologia foram utilizadas as técnicas histológicas de rotina (Behmer et al., 1976). A coloração foi realizada com Hematoxilina-eosina (HE) e tricrômio de Masson e a leitura das lâminas, utilizando microscopia óptica, foi realizada no Núcleo de Estudos sobre Endo e Ectoparasitos de Interesse Médico e Veterinário/Depto. de Biologia/IOC/FIOCRUZ.

\footnotetext{
* Apoio financeiro: CNPq/FIOCRUZ.

** Departamento de Biologia/Pavilhão Lauro Travassos/IOC/FIOCRUZ. Manguinhos, Av. Brasil, 4.365, Rio de Janeiro, Brasil. CEP 21045-900.

*** Acadêmica da Faculdade de Medicina Veterinária/Univ. Estácio de Sá.

${ }^{\star \star \star \star \star}$ Faculdade de Medicina Veterinária. Univ. Federal Rural do Rio de Janeiro.
} 
Na técnica de Punção-biópsia hepática, a histologia demostrou sucesso em 70 das coletas realizadas. Sete das amostras continham tecido orgânico proveniente do pulmão e do diafragma, caracterizando erro no procedimento de coleta.

Vinte e oito e 26 das amostras analisadas através da técnica de Punção-biópsia hepática e da técnica de Quatro Tamises resultaram positivas. Contudo, é importante indicar que na técnica de Punção-biópsia hepática existe a possibilidade de suspeita da enfermidade baseada na lesão observada, havendo, desta forma, diferença significativa no número de animais negativos entre as técnicas utilizadas (Tabela). Entretanto, na técnica de Punção-biópsia hepática, deve ser considerada a necessidade da utilização de pessoas treinadas no momento da coleta, para a contenção do animal e a retirada da amostra (Scherer et al., 1995); e na leitura do material devido à necessidade de diagnóstico diferencial (Betty e Markson 1954; Döbereiner et. al.,1969; 1976; Driemayer et. al.; 1991; Fowller, 1968; Hill, 1963; Loosmorem \& Markson, 1961; Méndez et. al.,1990; Tokarnia e Döbereiner, 1984; Tokarnia et. al., 1990). Esses fatores encarecem o resultado e tornam o trabalho árduo e desgastante, pelo que se reco-

\section{Referências bibliográficas}

ALCAINO, H. e APT, W. Algunos antecedentes sobre la fascioliasis animal y humana. Monogr Med Vet., v. 11, p. 14-29, 1989.

BARROS, C. S. L., METZDORF, L. L. e PEIXOTO, PV. Ocorrência de surtos de intoxicação por Seneciosp. (Compositae) em bovinos no Rio Grande do Sul. Pesq Vet Bras., v. 7, n. 4, p. 101-107, 1987.

BEHMEROA DETOLOSA, E. M. C. e DE FREITASNETO, AG. Manual de técnicas para histologia normal e patológica. São Paulo: Edart, 1976. $257 \mathrm{p}$.

BERNE MEA. Identificação e purificação de antígenos somáticos de formas adultas de Fasciola hepatica através de anticorpos monoclonais: ensaios de imunoproteção e imunodiagnóstico. Tese apresentada ao curso de Pós-Graduação em Parasitologia do Instituto de Ciências Biológicas da UFMG, 168 p, 1994.

BETTY, R. M. e MARKSON, L. M. Liver biopsy in the diagnosis of regwort (Senecio jacobea)poisoning in aherd of cattle. Vet Rec., v. 66, n. 28, p. 398-400, 1954.

DÖBEREINER J, TOKARNIA CH \& CANELLA CFC. Intoxicação por Cestrum levigatum Schelecht, a causa de mortandades em bovinos no Estado do Rio de Janeiro. Pesq Agrop Bras., v. 4, p. 165193, 1969.

DÖBEREINER, J., TOKARNIA. C. H., MONTEIRO, M. C. C., DACRUZ, L. C. H., CARVALHO, E. G. e FRIMO, A. T. Intoxicação de bovinos e ovinos em pasto Brachiaria decumbens contaminados por Phitomyces chartarum. Pesq Agrop Bras. Ser Vet., v.11, p. 87-94, 1976.

DRIEMAYER, D., BARROS, C. S. L. e PILATI, C. Seneciose em bovinos. Hora Veterinária, v. 10, n. 59, jan./fev. 1991. menda a utilização da técnica de Punção-biópsia somente nos trabalhos experimentais, onde seja necessária a manutenção dos animais e a retirada de amostras hepáticas do hospedeiro vertebrado, como acontece em trabalhos histopatológicos e histoquímicos.

Tabela 1: Resultado dos exames analisados, a partir de amostras coletadas em bovinos provenientes do município de Santa Vitória do Palmar, Rio Grande do Sul, Brasil, através da técnica de Punção-biópsia e de Quatro tamises para o diagnóstico da fasciolose hepática

\begin{tabular}{|c|c|c|c|c|}
\hline & \multicolumn{2}{|c|}{ Téc. De Punção-biópsia } & \multicolumn{2}{|c|}{ Téc. de Cuatro tamises } \\
\hline & $\begin{array}{l}\mathrm{N}^{\circ} \text { de animais } \\
\text { positivos }\end{array}$ & $\%$ & $\begin{array}{l}N^{\circ} \text { de animais } \\
\text { positivos }\end{array}$ & $\%$ \\
\hline Positivos• & 28 & 36,36 & 26 & 33,77 \\
\hline Suspeitos & 34 & 44,16 & 0 & 0 \\
\hline Negativos*• & 15 & 19,48 & 51 & 66,23 \\
\hline
\end{tabular}

- $p<0,05$ (teste de Fisher)

* Sete amostras constituiram erro de técnica

FOWLLER ME. Pyrrolizidine Alkaloid Poissoning in Calves. JAVMA., v. 152, n. 8, p. 1131-1137, 1968.

GIRÃO, E. S. e UENO, H. Técnica de quatro tamises para diagnóstico coprológico quantitativo da Fasciolose dos ruminantes. Pesq Agrop Bras., v. 20, p. 905-912, 1985.

HILL, K. R. Comment on the histological apperearences in serial liver biopsies and potmortem specimens. Vet Rec., v. 75, n. 19, p. 493495, 1963.

LOOSMOREM, R. M. e MARKSON, L. M. Poisoning of cattle by Brazilian groundnut meal. Vet Rec., v. 73, p. 813-814, 1961.

MÉNDEZ, M. C, CORRÊA, F. R., SCHILD, A. L. \& MARTZ, W. Intoxicação experimental por cinco espécies de Senecio em bovinos e aves. Pesq Vet Bras., v. 10, n. 3-4, p 63-69, 1990.

SCHERER, P. O., ABIDU, M., SANTOS, C. S., SILVA, P. B., BORDIN, E. e SERRA-FREIRE, N. M. Ocorrência e prevalência da Fasciola hepatica na Região dos Lagos, Estado do Rio de Janeiro. Parasitol al Dia, v. 19, n.102, p. 69-71, 1995.

SCHERER, P. O., PILE, E. A., SERRA-FREIRE, N. M. e VIDAL, G. Uso da Técnica de Punção-biópsia do fígado de ovinos, para diagnóstico histopatológico da fasciolose. Brazilian Journal of Veterinary Research and Animal Science,.v. 36, n.4, 1999. No prelo.

TOKARNIA, C. H. e DÖBEREINER, J. Intoxicação experimental por Senecio brasiliensis (Compositae) em bovinos. Pesq Vet Bras., v. 4, n. 2, p. 39-65, 1984.

TOKARNIA, C. H., GAYA, A., PEIXOTO, P. V., STOLF, L., CONSORTE, L. B. e DÖBEREINER, J. Intoxicação experimental por Senecio desiderabilis (Compositae) em bovinos. Pesq Vet Bras., v. 10, n. 1-2, p. 35-42,1990. 